\title{
Ultrastructural analysis of Lyophilized Human Spermatozoa
}

\author{
Renata de Lima Bossi ${ }^{1,2}$, Marcelo Cabral ${ }^{1}$, Monica Oliveira ${ }^{3}$, Sávia Lopes ${ }^{3}$, Rodrigo Hurtado ${ }^{1,2}$, Marcos Sampaio² \\ Selmo Geber ${ }^{1,2}$ \\ ${ }^{1}$ Department of Obstetrics and Gynaecology of the Medical School, Universidade Federal de Minas Gerais, Belo \\ Horizonte, MG, Brazil \\ ${ }^{2}$ ORIGEN, Center for Reproductive Medicine, Belo Horizonte, MG, Brazil \\ ${ }^{3}$ Faculty of Pharmacy, Universidade Federal de Minas Gerais, Belo Horizonte, MG, Brazil
}

These results were presented at the $35^{\text {th }}$ annual meeting of the European Society of Human Reproduction and Embryology

\begin{abstract}
Objective: Lyophilization is potentially more practical and cost-effective alternative for sperm preservation. However, there are no studies that evaluate the ultrastructure of human spermatozoa after lyophilization. Therefore, the aim of our study was to evaluate the ultrasctructure of lyophilized spermatozoa using Transmission Electron Microscopy.

Methods: From a total of 21 donated seminal samples, 30 aliquots were originated and divided into two aliquots so that one could have been submitted to cryopreservation/thaw and the other for lyophilization/rehydration. The liquefied aliquots were homogenized at room temperature. Samples assigned for cryopreservation were placed in straws and samples assigned for lyophilization were placed in the appropriate vials. Cryopreservation samples were placed at $-30 \mathrm{oC}$ for 30 minutes subsequently for 30 minutes at vapour phase and then plunged into liquid nitrogen. Lately, were warmed in water bath at $370 \mathrm{C}$ for 10 minutes followed by 10 minutes centrifugation. The pellet was resuspended and analysed in a Makler chamber. The semen vials assigned for lyophilization were loaded into a pre-fixed freeze-drying chamber. Following lyophilization, vials were removed from the freeze-drying chamber and kept at $40 \mathrm{C}$ until rehydration. TEM was performed after rehydration and thawing. Sperm samples were fixed, rinsed in buffer, post fixed and dehydration was carried out in escalating concentrations of alcohol solution, acetone and then, embedding in Epon resin. Ultrathin sections were stained and examined in a Transmission Electron Microscope.

Results: Analysis of sperm after freezing/thawing using Transmission Electron Microscopy showed lesions to the midpiece, with some mitochondria degeneration and random rupture of plasma membrane. In the head, we identified intact plasma membrane, nucleus and acrosome, as in the flagellum all main structures remained intact including the plasma membrane, the longitudinal columns of dense fibers and the semicircular fibers. Analysis by Transmission Electron Microscopy showed that spermatozoa heads had ruptured plasma membranes, absence of acrosomes, nuclei with heterogeneous and decompressed chromatin. Mitochondria were deteriorated in the midpiece. Longitudinal columns of dense fibers were absent in the flagellum. Axonemes, in cross-sections, were disrupted with disorganized structures.

Conclusions: To our knowledge, our study demonstrated, for the first time, the structure of the human spermatozoa after lyophilization using Transmission Electron Microscopy. The use of a fixed lyophilization protocol with media containing cryoprotectants might explain the damage to the structures. More studies are necessary to improve the results of sperm lyophilization. In the future, the use of lyophilization of spermatozoa might reduce the costs of fertility preservation, since there will be no need for storage space and transportation is simpler.
\end{abstract}

Keywords: lyophilization, human semen, cryopreservation, transmission electron microscopy

\section{INTRODUCTION}

Cryopreservation of human spermatozoa is a widely used approach for male fertility preservation in cancer patients, as well as in other non-malignant conditions. It is also frequently applied for patients who will undertake fertility treatments using donor semen, patients who need to store spermatozoa for non-medical reasons and for azoospermic patients. Patients who have undergone testicular or epididymal sperm extraction and wish to avoid repeated surgical procedures may also benefit from this technique (Anger et al., 2003).

There are a few different liquid nitrogen-based methods for sperm cryopreservation such as slow freezing (Royere et al., 1996; Isachenko et al., 2012; Nallella et al., 2004), vitrification (Isachenko et al., 2005; Kuwayama et al., 2005; Vajta \& Nagy, 2006; Aizpurua et al., 2017; Shah et al., 2019; O'Neill et al., 2019), cryopreservation in empty zona pellucida (Cohen et al., 1997; AbdelHafez et al., 2009) and Cell Sleepers (Endo et al., 2012; Coetzee et al., 2016; Herbemont et al., 2019) and Sperm VD (Berkovitz et al., 2018; Li et al., 2019). Mild harmful effects on sperm structure and function may occur, regardless of the utilized cryopreservation technique. Thermal shock may induce intra and extracellular ice crystals formation, besides osmotic shock caused by excessive dehydration, which may also promote sperm death. Furthermore, the use of liquid nitrogen imposes some operative drawbacks such as the considerable storage space needed, transportation difficulties, risk of cross contamination of the samples, and maintenance costs (Clarke, 1999; Bielanski et al., 2003; Morris, 2005; Gianaroli et al., 2012). Lyophilization of spermatozoa might be a helpful alternative to liquid nitrogen-based methods.

Lyophilization or freeze-drying is a sublimation process in which water goes from the solid directly to the gas phase, under high vacuum and low (below freezing point) temperature (Gianaroli et al., 2012). Since there is nearly no need for storage space and transportation is simpler and the cost is considerably reduced. Also, as lyophilization leads to virus inactivation, the risk of viral contamination is more likely eliminated (Unger et al., 2009). The feasibility of this method has been reported in animal studies, with live birth reports of viable offspring after intracytoplasmic sperm injection (ICSI) in mice (Wakayama \& Yanagimachi, 1998; Kusakabe et al., 2001; Ward et al., 2003; Kawase \& Suzuki, 2011; Kusakabe, 2019; Wakayama et al., 2017), rabbits (Liu et al., 2004; Mercati et al., 2020), rats (Hirabayashi et al., 2005; Hochi et al., 2008; Muneto \& Horiuchi, 2011), horse (Choi et al., 2011; Restrepo et al., 2019), other domestic animals and endangered species (Kaneko et al., 2014; Olaciregui et al., 2015; Anzalone et al., 2018; Lv et al., 2019).

In humans, only scarce data have been published so far. Kusakabe et al. (2008) described chromosome integrity on 
human sperm after lyophilization and injection into enucleated mouse oocytes. Gianaroli et al. (2012) unveiled that lyophilization of human sperm damages cell membranes, but it doesn't affect DNA's integrity and Zhu et al. (2016) observed head and tail membrane damage using scanning electron microscopy (SEM)

To our knowledge there are no published studies that evaluate the ultrastructure of human spermatozoa after Iyophilization and Transmission Electron Microscopy (TEM) is a valid tool for such evaluation. Therefore, the aim of our study was to evaluate the ultrasctructure of lyophilized spermatozoa using TEM.

\section{Material and methods}

A total of 21 healthy men who had been subjected to assisted reproductive technology (ART) treatment from August 2014 to August 2015 donated their remaining semen sample for this study. All donors read and signed an informed consent form. The study was approved by the Research Ethics Committee of the Universidade Federal de Minas Gerais (COEP/UFMG - No 743.984). Semen samples were classified as normozoospermic according to World Health Organization (WHO) criteria.

\section{Semen preparation}

After ejaculation, semen samples were allowed to liquefy at room temperature for 30 minutes and were classified for count, motility and morphology, according to the World Health Organization (WHO, 2010) criteria. After selection for ICSI, the remaining volume was divided into two aliquots: one for cryopreservation/lyophilization and the other for cryopreservation, as a control.

The liquefied aliquots were homogenized with Freeze Medium (Irvine Scientific, USA) or Sperm Freeze Solution (Vitrolife, Sweden), both at room temperature in 1:1 proportion. Samples assigned for cryopreservation were placed in 0,5 mL CryoBiosystem straws (IMV Technologies, France), and samples assigned for lyophilization were placed in the appropriate vials for freeze-drying.

\section{Cryopreservation and Thawing}

Cryopreservation samples were placed at $-30^{\circ} \mathrm{C}$ for 30 minutes subsequently for 30 minutes at vapour phase and then plunged into liquid nitrogen. Cryopreserved sperm samples (control) were warmed in water bath at $37^{\circ} \mathrm{C}$ for 10 minutes followed by 10 minutes centrifugation at 200 $\mathrm{G}$ with culture medium mHTF (Irvine Scientific, USA). The pellet was resuspended in $0,3 \mathrm{~mL}$ of $\mathrm{mHTF}$ and then analysed in a Makler chamber.

\section{Lyophilization and Rehydration}

The semen vials assigned for lyophilization were loaded into a freeze-drying chamber (ModulyoD-115, Thermo Fisher Scientific) for 24 hours where temperature was maintained at $-50^{\circ} \mathrm{C}$ and pressure at 50-100 $\mu$ bar. Following lyophilization, vials were removed from the freeze-drying chamber and kept at $4^{\circ} \mathrm{C}$ until rehydration with $0.5 \mathrm{~mL}$ of water for embryo transfer (Sigma, USA) and analysed by optical microscopy.

\section{Ultrastructural analysis}

Transmission Electron Microscopy was performed after completion of rehydration and thawing of lyophilized and frozen samples for ultrastructural analysis, at the Centro de Microscopia Eletrônica da Universidade Federal de Minas Gerais. Sperm samples were fixed for 24 hours at a $4^{\circ} \mathrm{C}$ temperature in a solution containing $2.5 \%$ glutaraldehyde, $2 \%$ paraformaldehyde, and $0.1 \mathrm{M}$ of phosphate buffer. After fixation, the specimens were rinsed in buffer, and post fixed in $2 \%$ osmium tetroxide $\left(\mathrm{OsO}_{4}\right)$ for 2 hours at room temperature. Dehydration was carried out in escalating concentrations of alcohol solution (35\%-100\%), followed by acetone and then, embedding in Epon resin. Ultrathin sections (50-60 nm) were stained with lead citrate (Reynolds Solution) and examined in a Tecnai G2-12 (SpiritBiotwin FEI - $120 \mathrm{kV}$ ) Transmission Electron Microscope, operating at $80 \mathrm{kV}$ (Oregon, USA).

\section{RESULTS}

From a total of 21 donated seminal samples, 30 aliquots were originated and divided into two aliquots so that one could have been submitted to cryopreservation/thaw and the other for lyophilization/rehydration. The patients' age ranged from 32 to 56 years ( $38.7 \pm 5.2$ years) and the sexual abstinence ranged from 3 to 10 days $(3.7 \pm 2.1$ days). Seminal volume ranged from 1.5 to $4.6 \mathrm{~mL}(2.6 \pm 1.5$ $\mathrm{mL}), \mathrm{pH}$ ranged from 7.5 to $8.5(8.02 \pm 0.06)$. Initial sperm concentration ranged from $20 \times 10^{6}$ to the maximum of $176 \times 10^{6}\left(75.74 \times 10^{6} \pm 27.2\right)$. Progressive motility ranged from $28 \%$ to $89 \%(59 \% \pm 18.52)$; non-progressive motility ranged from 0 to $20 \%(26.05 \% \pm 6.22)$. Normal morphology ranged from $4 \%$ to $18 \%(7.95 \% \pm 2.1 \%)$. Normal chromatin ranged from $70 \%$ to $98 \%(90.3 \% \pm 5.3 \%)$.

Fresh spermatozoa analysis using Transmission Electron Microscopy showed intact heads and plasma membranes, compact and homogeneous chromatin in the nuclei, physiological vacuoles, acrosomes with visible internal and external membranes and normal subacrosomic spaces. In the midpiece, mitochondria and external dense fibers were observed. It was also possible to identify the semicircular fibers and two longitudinal columns of dense fibers, alongside with the plasma membrane, constituting the flagellum. Transverse sections of the axonemes from the middle piece, showed the plasma membrane, mitochondria sheath involving the outer dense fibers, nine pairs of peripheral microtubules plus two pairs of central microtubules. The transverse sections of axonemes from the flagellum region showed plasma membrane involving the sheath of dense fibers, nine pairs of peripheral microtubules plus two pairs of central microtubules (Figure 1).

Analysis of sperm after freezing/thawing showed concentration ranging from $5.6 \times 10^{6}$ to $140 \times 10^{6}$ $\left(44.75 \times 10^{6} \pm 35.08\right)$, progressive motility ranging from 1 to $58 \%(23.6 \% \pm 14.45)$ and non-progressive motility ranged from 2 to $20 \%(10,65 \% \pm 4.90)$. Transmission Electron Microscopy showed lesions to the midpiece, with some mitochondria degeneration and random rupture of plasma membrane. In the head, we identified intact plasma membrane, nucleus and acrosome, as in the flagellum all main structures remained intact including the plasma membrane, the longitudinal columns of dense fibers and the semicircular fibers (Figure 2).

After rehydration, lyophilized samples were initially analysed using optical microscopy and all spermatozoa were immotile. Analysis by Transmission Electron Microscopy showed that spermatozoa heads had ruptured plasma membranes, absence of acrosomes, nuclei with heterogeneous and decompressed chromatin. Mitochondria were deteriorated in the midpiece. Longitudinal columns of dense fibers were absent in the flagellum. Axonemes, in cross-sections, were disrupted with disorganized structures (Figure 3 ).

\section{DISCUSSION}

This study observed the ultrastructure of the lyophilized human spermatozoa using Transmission Electron Microscopy, with emphasis on the analysis of structural integrity of the plasma membrane, mitochondria, acrosome, mid piece, axoneme, nucleus and its chromatin, and flagellum, after lyophilization using a fixed protocol. 


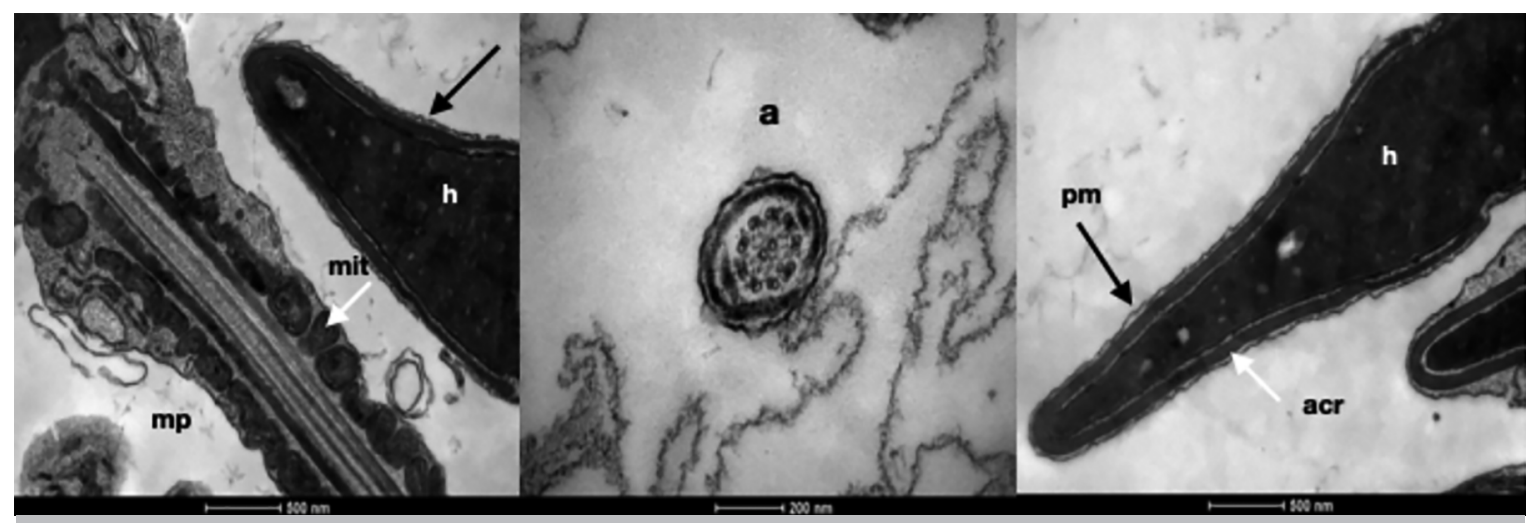

Figure 1. Transmission Electron Microscopy of Fresh Sperm: structure and organelles intact. a: axoneme, acr: acrossome, h: head, mit: mitochondria, mp: midpiece, pm: plasma membrane.

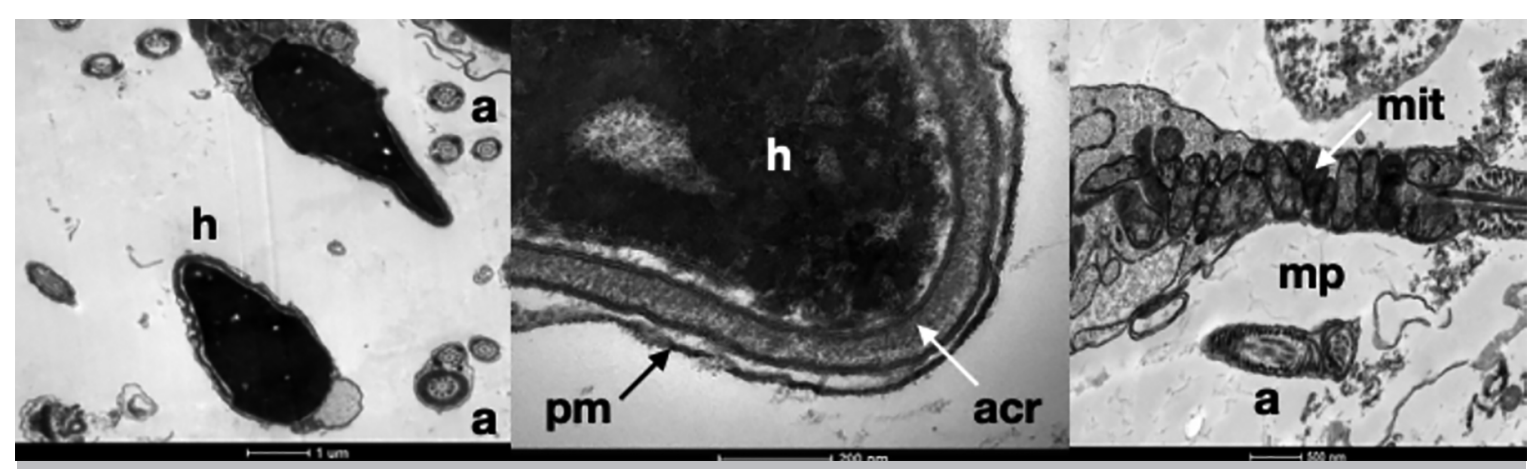

Figure 2. Transmission Electron Microscopy of Cryopreserved Sperm: structure intact. a: axoneme, acr: acrossome, $\mathrm{h}$ : head, mit: mitochondria, $\mathrm{mp}$ : midpiece, pm: plasma membrane.

When compared to the frozen/thawed samples (control group), the fresh samples showed higher concentration $\left(75.74 \times 10^{6} \times 44.75 \times 10^{6}\right)$, and higher sperm progressive and non-progressive motility, $59 \% \times 23.6 \%$ and $26.05 \% \times$ $10.65 \%$, respectively. All these occurred within the expected range, and can be explained by the fact that cryopreservation leads to a decrease in oxidative phosphorylation (Hallak et al., 2000; Wang et al., 2003). The morphological analysis using Transmission Electron Microscopy demonstrated that frozen spermatozoa maintained intact plasma, nucleus, mid piece and flagellum structures.

The observed effect of lyophilization on human sperm was the total loss of motility and severe injury to the cells with decreased viability after rehydration. These results are similar to those described by Kusakabe et al. (2008) and Gianaroli et al. (2012) who observed that although spermatozoa were physiologically dead, they still had intact DNA. Zhu et al. (2016) also observed head and tail membrane damage, however the authors used only SEM. These findings could be explained by the fact that glycerol-containing media cannot be lyophilized successfully, which probably led to the vast organelle damage (Alcay et al., 2015). Freeze Medium (Irvine Scientific) used in our study is rich in low density lipoprotein, whose function is to protect plasma membrane's phospholipids against freezing and thawing damage. Samples that were lyophilized using this medium did not form a good lyophile, probably due to the presence of glycerol, and consequently suffered membrane rupture, despite the presence of low-density lipoproteins. These observed lesions were probably caused during the dehydration process, since in control frozen samples the structures and organelles remained intact.
Several solutions for lyophilization have been used to try to maintain sperm integrity, such as albumin (Wakayama \& Yanagimachi, 1998), EDTA (Kaneko \& Nakagata, 2006) trehalose (McGinnis et al., 2005), combinations of disaccharides such as lactose, trehalose and sucrose (Garcia et al., 2014). Kaneko et al. (2003) observed that when lyophilization was performed in a slightly alkaline solution ( $\mathrm{pH} \mathrm{8.0)}$ the integrity of the chromosomes was maintained, and embryo development was better. Although we used freeze mediums with a lower $\mathrm{pH}$ (7.4) we cannot state that this particularly caused to the high structural damage observed (Kaneko et al., 2003).

As we used a constant pressure of 0.05 mbar throughout the lyophilization process, we consider this might have contributed to the sperm structural damage observed in our study. Kawase et al. (2007) recommend a primary pressure of 0.37 mbar for lyophilization of mouse spermatozoa. They suggested this value based on the observation of sperm DNA integrity and embryo development in vivo and in vitro. Kusakabe et al. (2008) used the pressure at 22 to $42 \times 10^{-3}$ mbar for 4 hours to lyophilize samples of human semen. Gianaroli et al. (2012) used a similar pressure ( $\left.22 \times 10^{-3} \mathrm{mbar}\right)$ overnight to lyophilize human semen.

The lyophilization process in our study lasted 24 hours. This long time interval may have led to excessive dehydration of the sample, and consequent damage to the sperm. Kwon et al. (2004) observed that increasing the time interval from 4 to 24 hours of lyophilization of pig spermatozoa gradually reduced embryonic development after fertilization. However, Gianaroli et al. (2012) lyophilized human semen overnight and obtained satisfactory results.

The storage temperature of the freeze-dried samples in our study ranged from $4^{\circ}$ to $8^{\circ} \mathrm{C}$ and was similar to what 


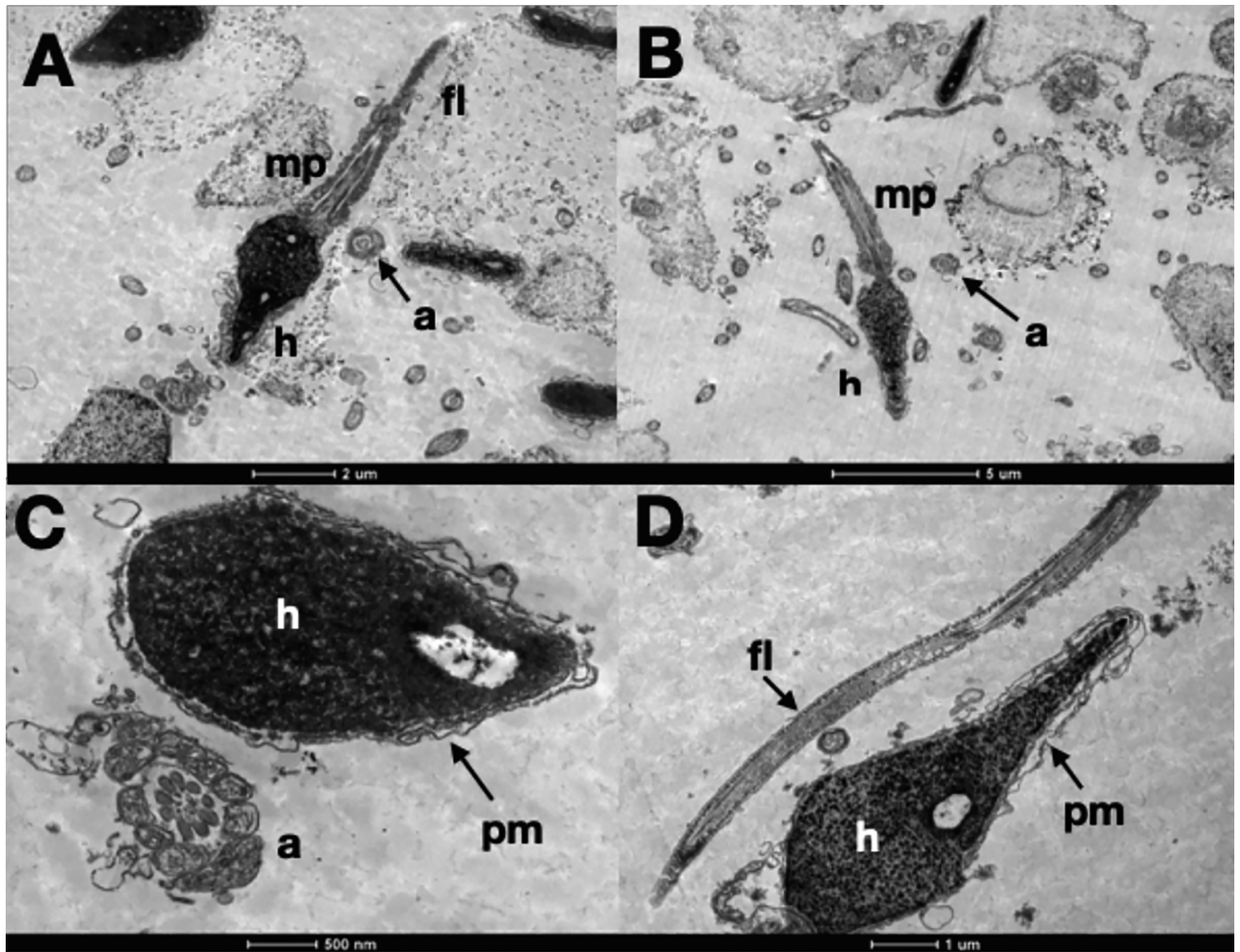

Figure 3. Transmission Electron Microscopy of Cryopreserved Sperm. A and C: Lyophilized in Sperm Freeze Medium: ruptured plasma membrane in head and flagellum, heterogeneous chromatin. Axoneme degenerated. B and D: Lyophilized in Freeze Medium: nuclei severed compromised, ruptured plasma membrane in head and flagellum, heterogeneous chromatin. a: axoneme, fl: flagellum, h: head, mp: midpiece, pm: plasma membrane.

others had described. Wakayama \& Yanagimachi (1998) demonstrated that refrigerated lyophilized mice sperm could generate healthy offspring after ICSI). Kaneko \& Nakagata (2005) kept lyophilized murine sperm at $4^{\circ} \mathrm{C}$ and observed normal DNA structure. Gianaroli et al. (2012) stored human sperm samples after lyophilization at $4^{\circ} \mathrm{C}$ and also observed no damage to sperm DNA.

Liu et al. (2004) studied the behaviour of lyophilized rabbit sperm in EGTA, $\mathrm{NaCl}$ and Tris- $\mathrm{HCl}$ buffered medium using Scanning Electron Microscopy. They observed that many spermatozoa had broken or absent flagellum. Plasma membrane was ruptured in flagellum region and midpiece, alongside damaged axoneme structure, in accordance with our findings using Transmission Electron Microscopy. Wakayama \& Yanagimachi (1998) freeze-dried mice sperm using culture media CZB and DMEM, and observed through Scanning Electron Microscopy, the following alterations in the sperm head: absence of the main segment of the acrosome, internal and external membranes of the acrosome ruptured or even absent. The nucleus remained intact and live offspring were obtained by performing ICSI with rehydrated spermatozoa. In our study we observed a decondensed chromatin, plasma membrane rupture and absent acrosome.

Martins et al. (2007) used three different solutions to lyophilize bovine sperm. The plasma membrane of spermatozoa was damaged in the lyophilization process independent of the medium used however the acrosome and mitochondria structures remained intact in all three media used. Differently from the study by Martins et al. (2007), the present study showed extensive damage to these structures.

Lyophilization might be an alternative to the use of liquid nitrogen for sperm cryopreservation since storage and transportation are a lot simpler, and the costs involved will be reduced. Also, as lyophilization is associated to virus inactivation, the risk of viral contamination will be virtually eliminated (Unger et al., 2009). More studies are needed to implement lyophilization as a routine technique in assisted reproduction clinics. Parameters such as temperature, pressure, time and lyophilization medium should be optimized to obtain lyophilized samples with intact DNA. After defining the best parameters, chromosomal viability tests should be performed.

To our knowledge, our study demonstrated, for the first time, the structure of the human spermatozoa after lyophilization using Transmission Electron Microscopy. The use of a fixed lyophilization protocol with media containing cryoprotectants might explain the damage to the structures. More studies are necessary to improve the results of sperm lyophilization. In the future, the use of lyophilization of spermatozoa might reduce the costs of fertility preservation, since there will be no need for storage space and transportation is simpler. 


\section{FUNDING}

Professor Selmo Geber has a Grant from the Fundação de Amparo à Pesquisa do Estado de Minas Gerais (FAPEMIG)

\section{CONFLICT OF INTEREST}

The authors have no potential conflicts of interest in the submitting manuscript.

\section{Corresponding author:}

Selmo Geber

1) Department of Obstetrics and Gynaecology

Medical School

Universidade Federal de Minas Gerais

2) ORIGEN, Center for Reproductive Medicine

Belo Horizonte - MG - Brazil

ORCID 0000-0001-7078-5438

E-mail: selmogeber@origen.com.br

\section{REFERENCES}

AbdelHafez F, Bedaiwy M, El-Nashar SA, Sabanegh E, Desai $\mathrm{N}$. Techniques for cryopreservation of individual or small numbers of human spermatozoa: a systematic review. Hum Reprod Update. 2009;15:153-64. PMID: 19109313 DOI: $10.1093 /$ humupd/dmn061

Aizpurua J, Medrano L, Enciso M, Sarasa J, Romero A, Fernández MA, Gómez-Torres MJ. New permeable cryoprotectant-free vitrification method for native human sperm. Hum Reprod. 2017;32:2007-15. PMID: 28938751 DOI: $10.1093 /$ humrep/dex281

Alcay S, Berk Toker M, Gokce E, Ustuner B, Tekin Onder N, Sagirkaya H, Nur Z, Kemal Soylu M. Successful ram semen cryopreservation with lyophilized egg yolk-based extender. Cryobiology. 2015;71:329-33. PMID: 26277039 DOI: 10.1016/j.cryobiol.2015.08.008

Anger JT, Gilbert BR, Goldstein M. Cryopreservation of sperm: indications, methods and results. J Urol. 2003;170:1079-84. PMID: 14501696 DOI: 10.1097/01. ju.0000084820.98430.b8

Anzalone DA, Palazzese L, Iuso D, Martino G, Loi P. Freezedried spermatozoa: An alternative biobanking option for endangered species. Anim Reprod Sci. 2018;190:85-93. PMID: 29397252 DOI: 10.1016/j.anireprosci.2018.01.010

Berkovitz A, Miller N, Silberman M, Belenky M, Itsykson P. A novel solution for freezing small numbers of spermatozoa using a sperm vitrification device. Hum Reprod. 2018;33:1975-83. PMID: 30285105 DOI: 10.1093/humrep/dey304

Bielanski A, Bergeron H, Lau PC, Devenish J. Microbial contamination of embryos and semen during long term banking in liquid nitrogen. Cryobiology. 2003;46:146-52. PMID: 12686204 DOI: $10.1016 /$ S0011-2240(03)00020-8

Choi YH, Varner DD, Love CC, Hartman DL, Hinrichs K. Production of live foals via intracytoplasmic injection of lyophilized sperm and sperm extract in the horse. Reproduction. 2011;142:529-38. PMID: 21846810 DOI: 10.1530/ REP-11-0145

Clarke GN. Sperm cryopreservation: is there a significant risk of cross-contamination? Hum Reprod. 1999;14:29413. PMID: 10601075 DOI: 10.1093/humrep/14.12.2941
Coetzee K, Ozgur K, Berkkanoglu M, Bulut H, Isikli A. Reliable single sperm cryopreservation in Cell Sleepers for azoospermia management. Andrologia. 2016;48:203-10. PMID: 25988980 DOI: 10.1111/and.12434

Cohen J, Garrisi GJ, Congedo-Ferrara TA, Kieck KA, Schimmel TW, Scott RT. Cryopreservation of single human spermatozoa. Hum Reprod. 1997;12:994-1001. PMID: 9194653 DOI: $10.1093 /$ humrep/12.5.994

Endo Y, Fujii Y, Shintani K, Seo M, Motoyama H, Funahashi $\mathrm{H}$. Simple vitrification for small numbers of human spermatozoa. Reprod Biomed Online. 2012;24:301-7. PMID: 22285239 DOI: $10.1016 /$ j.rbmo.2011.11.016

Garcia A, Gil L, Malo C, Martinez F, Kershaw-Young C, de Blas I. Effect of different disaccharides on the integrity and fertilising ability of freeze-dried boar spermatozoa: a preliminary study. Cryo Letters. 2014;35:277-85. PMID: 25282494

Gianaroli L, Magli MC, Stanghellini I, Crippa A, Crivello AM, Pescatori ES, Ferraretti AP. DNA integrity is maintained after freeze-drying of human spermatozoa. Fertil Steril. 2012;97:1067-73.e1. PMID: 22386843 DOI: 10.1016/j. fertnstert.2012.02.014

Hallak J, Mahran A, Chae J, Agarwal A. The effects of cryopreservation on semen from men with sarcoma or carcinoma. J Assist Reprod Genet. 2000;17:218-21. PMID: 10955246 DOI: $10.1023 / A: 1009443901307$

Herbemont C, Mnallah S, Grynberg M, Sifer C. Étude prospective comparant plusieurs techniques de congélation de faibles nombres de spermatozoïdes humains [Prospective comparison of different techniques for cryopreservation of small numbers of human spermatozoa]. Gynecol Obstet Fertil Senol. 2019;47:797-801. French. PMID: 31593817 DOI: $10.1016 /$ j.gofs.2019.09.014

Hirabayashi M, Kato M, Ito J, Hochi S. Viable rat offspring derived from oocytes intracytoplasmically injected with freeze-dried sperm heads. Zygote. 2005;13:79-85. PMID: 15984166 DOI: $10.1017 / S 096719940500300 X$

Hochi S, Watanabe K, Kato M, Hirabayashi M. Live rats resulting from injection of oocytes with spermatozoa freeze-dried and stored for one year. Mol Reprod Dev. 2008;75:890-4. PMID: 17926349 DOI: 10.1002/ mrd. 20825

Isachenko $\mathrm{V}$, Isachenko $\mathrm{E}$, Montag $\mathrm{M}$, Zaeva $\mathrm{V}$, Krivokharchenko I, Nawroth F, Dessole S, Katkov II, van der Ven H. Clean technique for cryoprotectant-free vitrification of human spermatozoa. Reprod Biomed Online. 2005;10:350-4. PMID: 15820041 DOI: 10.1016/S1472-6483(10)61795-6

Isachenko E, Mallmann P, Rahimi G, Risopatròn J, Schulz M, Isachenko V, Sànchez R. Vitrification Technique - New Possibilities for Male Gamete Low-Temperature Storage. In: Katkov II, ed. Current Frontiers in Cryobiology. London: IntechOpen; 2012. p. 41-76.

Kaneko T, Whittingham DG, Yanagimachi R. Effect of $\mathrm{pH}$ value of freeze-drying solution on the chromosome integrity and developmental ability of mouse spermatozoa. Biol Reprod. 2003;68:136-9. PMID: 12493705 DOI: 10.1095/ biolreprod.102.008706 
Kaneko T, Nakagata N. Relation between storage temperature and fertilizing ability of freeze-dried mouse spermatozoa. Comp Med. 2005;55:140-4. PMID: 15884775

Kaneko T, Nakagata N. Improvement in the long-term stability of freeze-dried mouse spermatozoa by adding of a chelating agent. Cryobiology. 2006;53:279-82. PMID: 16870171 DOI: $10.1016 /$ j.cryobiol.2006.06.004

Kaneko $T$, Ito $H$, Sakamoto $H$, Onuma $M$, Inoue-Murayama M. Sperm preservation by freeze-drying for the conservation of wild animals. PLoS One. 2014;9:e113381. PMID: 25409172 DOI: 10.1371/journal.pone.0113381

Kawase Y, Hani T, Kamada N, Jishage K, Suzuki H. Effect of pressure at primary drying of freeze-drying mouse sperm reproduction ability and preservation potential. Reproduction. 2007;133:841-6. PMID: 17504927 DOI: 10.1530/ REP-06-0170

Kawase $\mathrm{Y}$, Suzuki $\mathrm{H}$. A study on freeze-drying as a method of preserving mouse sperm. J Reprod Dev. 2011;57:17682. PMID: 21551975 DOI: 10.1262/jrd.10-199E

Kusakabe H, Szczygiel MA, Whittingham DG, Yanagimachi R. Maintenance of genetic integrity in frozen and freeze-dried mouse spermatozoa. Proc Natl Acad Sci U S A. 2001;98:13501-6. PMID: 11707598 DOI: 10.1073/ pnas. 241517598

Kusakabe $H$, Yanagimachi $R$, Kamiguchi $Y$. Mouse and human spermatozoa can be freeze-dried without damaging their chromosomes. Hum Reprod. 2008;23:233-9. PMID: 18056060 DOI: $10.1093 /$ humrep/dem 252

Kusakabe $\mathrm{H}$. Production of mouse fetuses using spermatozoa exposed temporarily to high temperature or continuously to room temperature after freeze-drying in $\mathrm{Na+-}$ free/K+-rich EGTA buffer. Cryobiology. 2019;87:105-9. PMID: 30682339 DOI: 10.1016/j.cryobiol.2019.01.010

Kuwayama M, Vajta G, Kato O, Leibo SP. Highly efficient vitrification method for cryopreservation of human oocytes. Reprod Biomed Online. 2005;11:300-8. PMID: 16176668 DOI: $10.1016 / S 1472-6483(10) 60837-1$

Kwon IK, Park KE, Niwa K. Activation, pronuclear formation, and development in vitro of pig oocytes following intracytoplasmic injection of freeze-dried spermatozoa. Biol Reprod. 2004;71:1430-6. PMID: 15215192 DOI: 10.1095/ biolreprod.104.031260

Li Y, Ma B, Yu P. Evaluation and improvement of the novel method for vitrification of a few human sperms. Eur J Obstet Gynecol Reprod Biol. 2019;240:74-9. PMID: 31234060 DOI: $10.1016 /$ j.ejogrb.2019.06.021

Liu JL, Kusakabe H, Chang CC, Suzuki H, Schmidt DW, Julian M, Pfeffer R, Bormann CL, Tian XC, Yanagimachi R, Yang $X$. Freeze-dried sperm fertilization leads to full-term development in rabbits. Biol Reprod. 2004;70:1776-81. PMID: 14960482 DOI: 10.1095/biolreprod.103.025957

Lv C, Wu G, Hong Q, Quan G. Spermatozoa Cryopreservation: State of Art and Future in Small Ruminants. Biopreserv Biobank. 2019;17:171-82. PMID: 30499684 DOI: 10.1089/bio.2018.0113
Martins CF, Báo SN, Dode MN, Correa GA, Rumpf R. Effects of freeze-drying on cytology, ultrastructure, DNA fragmentation, and fertilizing ability of bovine sperm. Theriogenology. 2007;67:1307-15. PMID: 17383718 DOI: 10.1016/j. theriogenology.2007.01.015

McGinnis LK, Zhu L, Lawitts JA, Bhowmick S, Toner M, Biggers JD. Mouse sperm desiccated and stored in trehalose medium without freezing. Biol Reprod. 2005;73:627-33. PMID: 15930320 DOI: 10.1095/biolreprod.105.042291

Mercati F, Domingo P, Pasquariello R, Dall'Aglio C, Di Michele A, Forti K, Cocci P, Boiti C, Gil L, Zerani M, Maranesi $M$. Effect of chelating and antioxidant agents on morphology and DNA methylation in freeze-drying rabbit (Oryctolagus cuniculus) spermatozoa. Reprod Domest Anim. 2020;55:29-37. PMID: 31626708 DOI: 10.1111/ rda. 13577

Morris GJ. The origin, ultrastructure, and microbiology of the sediment accumulating in liquid nitrogen storage vessels. Cryobiology. 2005;50:231-8. PMID: 15925575 DOI: 10.1016/j.cryobiol.2005.01.005

Muneto T, Horiuchi T. Full-term Development of Hamster Embryos Produced by Injecting Freeze-dried Spermatozoa into Oocytes. J Mamm Ova Res. 2011;28:32-9. DOI: 10.1274/jmor.28.32

Nallella KP, Sharma RK, Allamaneni SS, Aziz N, Agarwal A. Cryopreservation of human spermatozoa: comparison of two cryopreservation methods and three cryoprotectants. Fertil Steril. 2004;82:913-8. PMID: 15482768 DOI: $10.1016 /$ j.fertnstert.2004.02.126

O’Neill HC, Nikoloska M, Ho H, Doshi A, Maalouf W. Improved cryopreservation of spermatozoa using vitrification: comparison of cryoprotectants and a novel device for long-term storage. J Assist Reprod Genet. 2019;36:171320. PMID: 31273587 DOI: $10.1007 / s 10815-019-01505-x$

Olaciregui M, Luño V, Gonzalez N, De Blas I, Gil L. Freezedried dog sperm: Dynamics of DNA integrity. Cryobiology. 2015;71:286-90. PMID: 26247315 DOI: $10.1016 /$ j.cryobiol.2015.08.001

Restrepo G, Varela E, Duque JE, Gómez JE, Rojas M. Freezing, Vitrification, and Freeze-Drying of Equine Spermatozoa: Impact on Mitochondrial Membrane Potential, Lipid Peroxidation, and DNA Integrity. J Equine Vet Sci. 2019;72:8-15. PMID: 30929788 DOI: 10.1016/j. jevs.2018.10.006

Royere D, Barthelemy C, Hamamah S, Lansac J. Cryopreservation of spermatozoa: a 1996 review. Hum Reprod Update. 1996;2:553-9. PMID: 9111188 DOI: 10.1093/humupd/2.6.553

Shah D, Rasappan, Shila, Gunasekaran K. A simple method of human sperm vitrification. MethodsX. 2019;6:2198204. PMID: 31667120 DOI: 10.1016/j.mex.2019.09.022

Unger U, Poelsler G, Modrof J, Kreil TR. Virus inactivation during the freeze-drying processes as used for the manufacture of plasma-derived medicinal products. Transfusion. 2009;49:1924-30. PMID: 19453977 DOI: $10.1111 / j .1537-$ 2995.2009.02218.x 
Vajta G, Nagy ZP. Are programmable freezers still needed in the embryo laboratory? Review on vitrification. Reprod Biomed Online. 2006;12:779-96. PMID: 16792858 DOI: 10.1016/S1472-6483(10)61091-7

Wakayama S, Kamada Y, Yamanaka K, Kohda T, Suzuki H, Shimazu T, Tada MN, Osada I, Nagamatsu A, Kamimura S, Nagatomo H, Mizutani E, Ishino F, Yano S, Wakayama $T$. Healthy offspring from freeze-dried mouse spermatozoa held on the International Space Station for 9 months. Proc Natl Acad Sci U S A. 2017;114:5988-93. PMID: 28533361 DOI: $10.1073 /$ pnas. 1701425114

Wakayama T, Yanagimachi R. Development of normal mice from oocytes injected with freeze-dried spermatozoa. Nat Biotechnol. 1998;16:639-41. PMID: 9661196 DOI: 10.1038/nbt0798-639
Wang X, Sharma RK, Sikka SC, Thomas AJ Jr, Falcone T, Agarwal A. Oxidative stress is associated with increased apoptosis leading to spermatozoa DNA damage in patients with male factor infertility. Fertil Steril. 2003;80:531-5. PMID: 12969693 DOI: $10.1016 /$ S0015-0282(03)00756-8

Ward MA, Kaneko T, Kusakabe H, Biggers JD, Whittingham DG, Yanagimachi R. Long-term preservation of mouse spermatozoa after freeze-drying and freezing without cryoprotection. Biol Reprod. 2003;69:2100-8. PMID: 12930716 DOI: 10.1095/biolreprod.103.020529

WHO World Health Organization. WHO laboratory manual for the Examination and processing of human semen. 5th ed. Geneva: WHO; 2010.

Zhu WJ, Li J, Xiao LJ. Changes on membrane integrity and ultrastructure of human sperm after freeze-drying. J Reprod Contracep 2016;27:76-81 